\title{
Association of Body Compositions and Bone Mineral Density in Chinese Children and Adolescents: Compositional Data Analysis
}

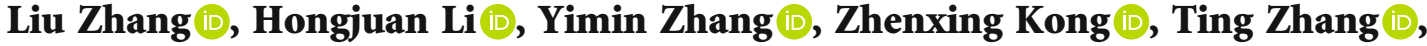 \\ and Zhaohua Zhang iD \\ School of Sport Science/Key Laboratory of the Ministry of Education of Exercise and Physical Fitness, Beijing Sport University, \\ Beijing 100084, China \\ Correspondence should be addressed to Hongjuan Li; janerobin@126.com
}

Received 28 June 2021; Revised 22 September 2021; Accepted 15 October 2021; Published 1 November 2021

Academic Editor: Ali Khani jeihooni

Copyright (c) 2021 Liu Zhang et al. This is an open access article distributed under the Creative Commons Attribution License, which permits unrestricted use, distribution, and reproduction in any medium, provided the original work is properly cited.

\begin{abstract}
The purpose of this study was to investigate the relationship between body compositions and bone mineral density (BMD) and the effect of composition substitution among Chinese children and adolescents without the influence of multicollinearity. A dualenergy X-ray absorptiometry scan was used to determine the amount of truncal fat (TF), nontruncal fat (NTF), fat-free mass (FFM), and BMD. The compositional data analysis and the compositional proportional substitution analysis were conducted to determine the effect of each part of body compositions on BMD and its substitution effects. Four hundred sixty-six (466) (boys: 51.9\%) participants completed this cross-sectional study. For girls, in the overweight group, the relationship between TF and the BMD was positive $(\beta=2.943 e-01, p=0.006)$ while the NTF showed the opposite trend $(\beta=-2.358 e-01, p=0.009)$. When $4 \%$ NTF or FFM was substituted by TF, the BMD increased by about 0.1 and 0.05 units $(p<0.05)$, respectively. For boys, the association between FFM and BMD was statistically positive $(\beta=4.091 e-02, p=0.0001)$. There was a positive correlation between TF and BMD $(\beta=7.963 e-02, p=0.036)$. But with the increase of $\mathrm{BMI}$, this correlation shifted in the opposite direction. In conclusion, compared to TF and NTF, FFM had a better protective effect on BMD, especially for boys. The risk of NTF accumulation on BMD was greater than that of TF accumulation. Compared with girls, boys were more sensitive to the amount of TF.
\end{abstract}

\section{Introduction}

Osteoporosis has become a global public health problem, causing a huge economic burden on society and reducing the quality of life. Peak bone mass is an important determinant of osteoporotic fracture risk, and adequate peak bone mass is associated with a reduced risk of osteoporotic fracture later in life [1]. Early childhood and adolescence are periods of rapid growth and bone mineral accumulation. At 18 , it can reach $90 \%$ of the individual's peak bone mass [2]. According to statistics, for every $10 \%$ increase in peak bone mass, the risk of osteoporotic fractures in the future will be reduced by $50 \%$ or the age of onset of osteoporosis will be delayed by 13 years [3].
Body composition is an important determinant of bone mineral density (BMD), which is mainly composed of two components: fat-free mass (FFM) and fat mass (FM) $[4,5]$. Numerous studies have explored the relationship between FFM and BMD and consistently shown a higher percentage of FFM associated with higher BMD because of the mechanical load and the load effect of weight-bearing exercise [6-8]. However, the relationship between FM and BMD is unclear, especially FM distribution and BMD. Traditionally, obesity is considered osteoprotective because excess adipose tissue has a weight-bearing effect on bones $[9,10]$. But after adjusting the body weight, some studies found a negative or null association between obesity and BMD $[11,12]$. Body fat distribution may also impact BMD. Cao and Pollock et al. 
pointed out that central obesity hurt bone density $[13,14]$, whereas Yi et al. believed hip fat was a protective factor [15]. There are limited studies addressing the relationship between fat distribution and BMD, especially in children and adolescents. These restricted prior observations suggest the need to explore the relationship between body composition components and BMD deeply and comprehensively.

Body fat rate or body mass index (BMI) is often used as a surrogate measure to reflect overweight and obesity; however, it cannot provide accurate information about the distribution of body fat (e.g., Android fat or Gynoid fat), nor can it distinguish whether body weight comes from fat, muscle, or bone. Compositional data comprises mutually exclusive and exhaustive parts that add up to a total of $100 \%$ or 1 [16]. For example, body compositions, consisting of truncal fat (TF), nontruncal fat (NTF), and FFM, have the characteristics of compositional data. Compositional data analysis (CoDA) is a statistical technique designed to properly model the association between compositional variables and the outcomes. Because of calculating the original absolute data to obtain the proportional structure, CoDA can further explain the relative information behind the absolute data. However, the constant sum constraint increases the multicollinearity among components $[17,18]$. To avoid the influence of multicollinearity and retain all the simplex measurement attributes, Egozcue et al. proposed the isometric log ratio (ILR), which was converted by constructing a standard orthogonal basis to make a compositional data map from the simplex space SD to the Euclidean space RD-1 [19]. Therefore, considering that each part of the body composition of children and adolescents is closely related, it is more reasonable to analyze the components as a whole under the CoDA.

Because of the critical period of bone growth in children and adolescents and the limited studies, understanding the impact of the constitution of body compositions and those distributions on bone health is important when planning strategies to prevent fractures and osteoporosis later in life. Therefore, by using CoDA (ILR transformation), which can avoid multicollinearity, this study is aimed at examining the association between body compositions (\%TF, \%NTF, and \%FFM) and BMD and the substitution effects when reallocating each part to others in Chinese children and adolescents.

\section{Materials and Methods}

2.1. Study Population and Design. The present study is a cross-sectional study, and the samples of school children are nonrepresentative. Recruitment of participants was performed on the school level. In the spring of 2010, the project leader contacted 16 schools in Beijing, China, and presented information about the study. Each student from the recruited classes received a letter with detailed information regarding the purposes and methods of the study, and the parents needed to sign the informed consent. Measurements took place between April and July 2010. The inclusion criteria of the research objects are as follows: (1) complete basic information (age and sex) at baseline and (2) complete all test items. Exclusion criteria: (1) participants with a history
TABLE 1: The geometric mean values of the three components of body compositions.

\begin{tabular}{lccc}
\hline & Boys & Girls & Total \\
\hline Body composition (\%) & & & \\
Truncal fat & 8.44 & 10.62 & 9.45 \\
Nontruncal fat & 10.64 & 13.82 & 12.09 \\
Fat-free mass & 80.92 & 75.56 & 78.46 \\
\hline
\end{tabular}

TABLE 2: Body composition variation matrix.

\begin{tabular}{lccc}
\hline & Truncal fat & Nontruncal fat & Fat-free mass \\
\hline Truncal fat & 0.0000000 & & \\
Nontruncal fat & 0.06036724 & 0.00000000 & \\
Fat-free mass & 0.46329548 & 0.33766990 & 0.00000000 \\
\hline
\end{tabular}

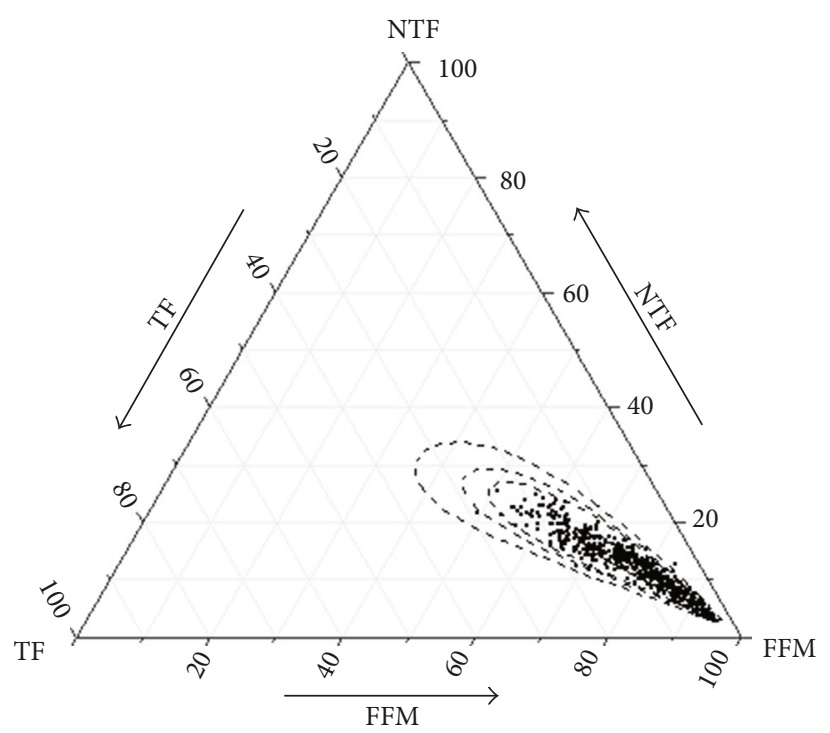

FIgURE 1: Relative information for body compositions is surrounded by $90 \%, 95 \%$, and $99 \%$ normal probability areas.

of diabetes, kidney disease, and other acute or chronic diseases and (2) limb disability. In total, 473 children aged 518 years voluntarily participated in this study, and the final effective sample size was 466 .

2.2. DXA Measurements. A dual-energy X-ray absorptiometry scan (DXA) was used to test all subjects' body compositions (TF, NTF, and FFM) and BMD (whole-body bone mineral density, upper-limb bone mineral density, thigh bone mineral density, trunk bone mineral density, and pelvic bone mineral density). The body mass was calculated as FFM + FM. We used the same DXA machine for all participants, calibrating machine drift daily with the standard synthetic phantom. Each measurement was taken by the welltrained operators.

2.3. Anthropometric Measurements. Height was assessed to the nearest $0.1 \mathrm{~cm}$ using a portable stadiometer by trained research staff. Body mass index (BMI) was calculated as body mass (in kilograms) divided by the square of height 


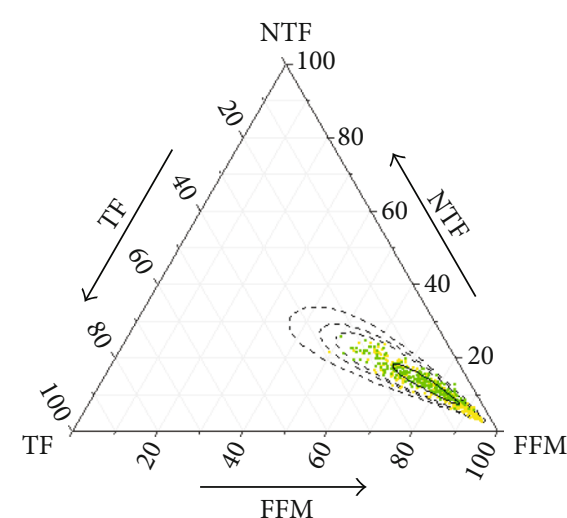

Gender

- (1)

= (2)

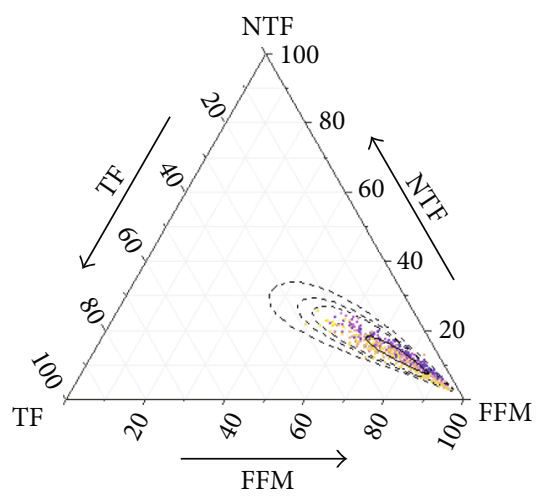

(c)

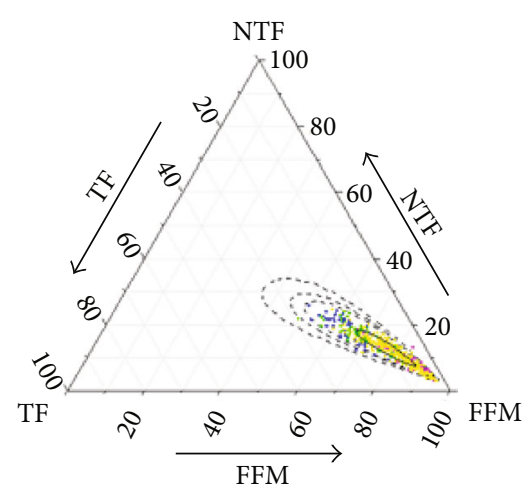

BMI group

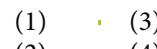

(2) $\cdot(4)$

(b)

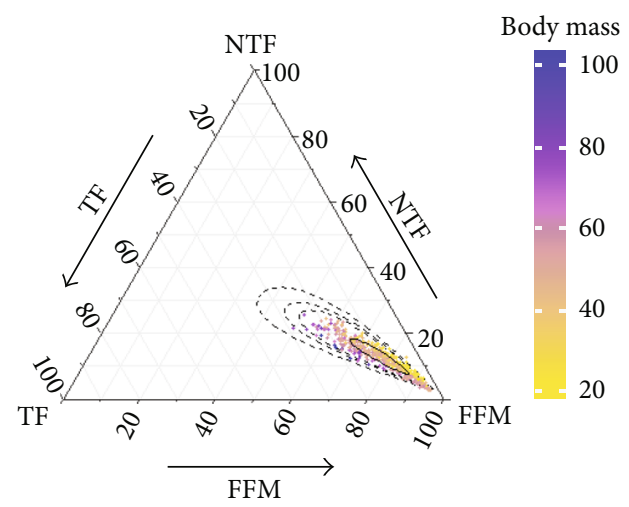

(d)

FIgURe 2: There is relative information of body compositions in different groups, surrounded by $90 \%$, $95 \%$, and $99 \%$ normal probability areas. (a) Gender group, 1: male and 2: female; (b) BMI group, 1: thin, 2: normal weight, 3: overweight, and 4: obesity; (c) age distribution; (d) body mass distribution.

(in meters). According to the BMI classification standard for overweight and obesity of school-age children and adolescents in China [20] and screening standard for malnutrition of school-age children and adolescents for a specific age and gender [21], subjects were divided into four groups: underweight, normal weight, overweight, and obesity.

2.4. Statistical Analysis. The first step of CoDA was to calculate the proportion of body compositions (\% $\mathrm{TF}, \% \mathrm{NTF}$, and $\%$ FFM). Then, the ratios were expressed as isometric logratio coordinates using ILR. Therefore, conventional descriptive statistics can be applied. The geometric mean was used as the central tendency of each component of the body composition, which can better show the center of the composition data point [22]. The ternary plot can visualize three components. The further away from the vertex indicates the smaller the proportion. The variation matrix was used to describe the discrete trend of CoDA. The more the value is connected to zero, the stronger the correlation between the corresponding two components. The compositional multiple regression model was used to analyze the relationship between body compositions and BMD of each part in different BMI groups; the compositional proportional substitution model was used to quantify the reallocation effects of body composition and BMD. Covariates include age and body mass. Analyses were performed in R 3.6.3 using the "compositions" package. $p$ values $<0.05$ were considered statistically significant.

\section{Results}

3.1. Descriptive Analysis. Four hundred sixty-six participants (51.93\% male) with completed data were included in this study. The mean age was 12.23 (3.13) years. Most (62.66\%) of the subjects were categorized as normal weight, and the proportion of overweight and obesity was $25.75 \%$. The geometric mean values of the three components of body composition are shown in Table 1.

3.2. Body Composition Variation Matrix. The variation matrix (Table 2) calculated the variance in the log ratio of two parts. The variation matrix value between TF and NTF was the smallest (0.06). The larger values between TF, 
TABLE 3: Multiple linear regression analysis of the relationship between body compositions and BMD (girls-compositional multiple regression model) $(n=225)$.

\begin{tabular}{|c|c|c|c|c|c|c|c|c|}
\hline & & $\operatorname{Adj} R^{2}$ & $\beta \mathrm{TF}$ & $\operatorname{Pr}(>|t|)$ & $\beta \mathrm{NTF}$ & $\operatorname{Pr}(>|t|)$ & $\beta$ FFM & $\operatorname{Pr}(>|t|)$ \\
\hline \multirow{5}{*}{ Whole-body BMD } & 1 & 0.8245 & $-1.060 e-04$ & 0.99820 & $-2.921 e-02$ & 0.69355 & $2.932 e-02$ & 0.60071 \\
\hline & 2 & 0.775 & $-4.895 e-02$ & 0.205 & $2.740 e-02$ & 0.508 & $2.154 e-02$ & 0.220 \\
\hline & 3 & 0.8756 & $2.943 e-01$ & $0.006405^{* *}$ & $-2.358 e-01$ & $0.009298^{* *}$ & $-5.857 e-02$ & 0.365043 \\
\hline & 4 & 0.8626 & $-2.462 e-03$ & 0.9904 & $2.030 e-02$ & 0.9450 & $-1.784 e-02$ & 0.9277 \\
\hline & Total & 0.8245 & $3.704 e-03$ & 0.889 & $-2.336 e-02$ & 0.446 & $1.966 e-02$ & 0.171 \\
\hline \multirow{5}{*}{ Upper limb BMD } & 1 & 0.7345 & $3.196 e-02$ & 0.43857 & $-2.949 e-02$ & 0.64873 & $-2.467 e-03$ & 0.95962 \\
\hline & 2 & 0.8119 & $-4.537 e-03$ & 0.86543 & $-1.998 e-02$ & 0.48813 & $2.452 e-02$ & $0.04554^{*}$ \\
\hline & 3 & 0.908 & $2.399 e-01$ & $0.000466^{* * *}$ & $-1.718 e-01$ & $0.00210^{* *}$ & $-6.807 e-02$ & 0.084868 \\
\hline & 4 & 0.8403 & $5.104 e-03$ & 0.97440 & $-3.061 e-01$ & 0.19892 & $3.010 e-01$ & 0.07048 \\
\hline & Total & 0.8368 & $3.647 e-02$ & 0.05973 & $-6.114 e-02$ & $0.00646^{* *}$ & $2.467 e-02$ & $0.0184^{*}$ \\
\hline \multirow{5}{*}{ Thigh BMD } & 1 & 0.8514 & $4.273 e-02$ & 0.5146 & $-1.276 e-01$ & 0.2235 & $8.489 e-02$ & 0.281 \\
\hline & 2 & 0.8643 & $-2.234 e-02$ & 0.6262 & $-3.463 e-02$ & 0.4830 & $5.697 e-02$ & $0.00697^{* *}$ \\
\hline & 3 & 0.933 & $3.561 e-01$ & $0.00128^{* *}$ & $-3.927 e-01$ & $9.23 e-05^{* * *}$ & $3.658 e-02$ & 0.5629 \\
\hline & 4 & 0.8292 & $-1.051 e-01$ & 0.6992 & $1.735 e-01$ & 0.6571 & $-6.832 e-02$ & 0.7929 \\
\hline & Total & 0.8649 & $9.711 e-02$ & $0.004807^{* *}$ & $-1.423 e-01$ & $0.000369^{* * *}$ & $4.519 e-02$ & $0.014680^{*}$ \\
\hline \multirow{5}{*}{ Trunk BMD } & 1 & 0.8199 & $1.304 e-02$ & 0.7656 & $-8.246 e-02$ & 0.2396 & $6.943 e-02$ & 0.1913 \\
\hline & 2 & 0.8022 & $2.831 e-02$ & 0.37543 & $-7.176 e-02$ & $0.0379^{*}$ & $4.344 e-02$ & $4.344 e-02$ \\
\hline & 3 & 0.9226 & $3.708 e-01$ & $0.000115^{* * *}$ & $-2.765 e-01$ & $0.000439^{* * *}$ & $-9.437 e-02$ & 0.077764 \\
\hline & 4 & 0.8669 & $1.569 e-01$ & 0.4310 & $-1.874 e-02$ & 0.9469 & $-1.382 e-01$ & 0.4682 \\
\hline & Total & 0.8502 & $4.448 e-02$ & 0.050306 & $-8.599 e-02$ & $0.00114^{* *}$ & $4.151 e-02$ & $0.000778^{* * *}$ \\
\hline \multirow{5}{*}{ Pelvic BMD } & 1 & 0.806 & $4.171 e-02$ & 0.5563 & $-1.487 e-01$ & 0.1911 & $1.070 e-01$ & 0.2115 \\
\hline & 2 & 0.8273 & $4.608 e-02$ & 0.3054 & $-9.748 e-02$ & $0.0448^{*}$ & $5.140 e-02$ & $0.0127^{*}$ \\
\hline & 3 & 0.8994 & $4.748 e-01$ & $0.000652^{* * *}$ & $-4.030 e-01$ & $0.000642^{* * *}$ & $-7.183 e-02$ & 0.361787 \\
\hline & 4 & 0.8021 & $1.463 e-01$ & 0.6273 & $-1.815 e-02$ & 0.9663 & $-1.282 e-01$ & 0.6569 \\
\hline & Total & 0.8486 & $1.002 e-01$ & $0.00267^{* *}$ & $-1.493 e-01$ & $0.000117^{* * *}$ & $4.909 e-02$ & $0.006233^{* *}$ \\
\hline
\end{tabular}

Note: 1 - underweight group $(n=25), 2$-normal-weight group $(n=160), 3$-overweight group $(n=25)$, and 4-obesity group $(n=15) ; p<0.05^{*} ; p<0.005^{* *}$ $; p<0.0005^{* * *}$.

NTF, and FFM (0.46 and 0.33) showed that there was a small correlation between fat mass and fat-free mass.

3.3. Results of Ternary Graphs for Different Groups. In this study, the body composition data was divided into three parts to be perfectly plotted in the ternary diagram. The distribution of data points in Figure 1 suggested that as the proportion of FFM increased (moving towards the peak of the triangle), the proportions of TF and NTF decreased more equally. Figure 2(a) shows the gender difference in the body compositions of the study subjects. Compared with girls, boys had a lower proportion of fat mass and a higher proportion of FFM. The points of children and adolescents in the underweight group and the normal-weight group were mostly concentrated at the tip of the triangle, composed of a lower proportion of adipose mass and a higher proportion of FFM (Figure 2(b)). With the increase of age and weight (Figures 2(c) and 2(d)), the proportion of TF showed an upward trend, and the proportion of NTF continued to decrease. Therefore, the next main analyses were discussed by gender, with age and body mass used as covariates.

3.4. Compositional Multiple Regression Analysis. Tables 3 and 4 present the association between the body compositions and BMD after adjusting for age and body mass. Table 3 shows the results of girls. For girls in the overweight group, the relationship between TF and the BMD of each part was positive, while the NTF showed the opposite trend $(p<0.01)$. For normal-weight children, NTF showed a significant negative correlation with trunk and pelvic BMD $(p<0.01)$. There was no significant correlation between FFM and whole-body BMD $(p=0.171)$. However, when we considered each part of the BMD, the relationships between FFM and BMD of upper limbs, thighs, trunk, and pelvis were statistically positive $(p<0.05)$.

For boys in Table 4, in addition to the BMD of the thigh, TF showed a strong positive correlation with the bone mineral density of the whole body, upper limbs, trunk, and 
TABLE 4: Multiple linear regression analysis of the relationship between body compositions and BMD (boys-compositional multiple regression model) $(n=241)$.

\begin{tabular}{|c|c|c|c|c|c|c|c|c|}
\hline & & $\operatorname{Adj} R^{2}$ & $\beta \mathrm{TF}$ & $\operatorname{Pr}(>|t|)$ & $\beta \mathrm{NTF}$ & $\operatorname{Pr}(>|t|)$ & $\beta \mathrm{FFM}$ & $\operatorname{Pr}(>|t|)$ \\
\hline \multirow{5}{*}{ Whole-body BMD } & 1 & 0.5917 & $8.401 e-02$ & 0.256432 & $-1.669 e-01$ & $0.044409^{*}$ & $8.286 e-02$ & $0.032967^{*}$ \\
\hline & 2 & 0.7837 & $7.963 e-02$ & $0.03602^{*}$ & $-1.067 e-01$ & $0.0107^{*}$ & $2.706 e-02$ & $0.0478^{*}$ \\
\hline & 3 & 0.823 & $7.971 e-02$ & 0.34717 & $-2.032 e-01$ & $0.0216^{*}$ & $1.235 e-01$ & $0.000205^{* * *}$ \\
\hline & 4 & 0.8811 & $-2.051 e-01$ & 0.09448 & $-1.182 e-02$ & 0.91017 & $2.169 e-01$ & $0.00141^{* *}$ \\
\hline & Total & 0.7836 & $6.460 e-02$ & $0.034039^{*}$ & $-1.055 e-01$ & $0.00144^{* *}$ & $4.091 e-02$ & $0.000108^{* * *}$ \\
\hline \multirow{5}{*}{ Upper-limb BMD } & 1 & 0.8226 & $4.317 e-02$ & 0.34878 & $-1.300 e-01$ & $0.01422^{*}$ & $8.683 e-02$ & $0.000887^{* * *}$ \\
\hline & 2 & 0.8377 & $0.00391^{* *}$ & $0.01089^{*}$ & $-8.974 e-02$ & $0.00391^{* *}$ & $1.774 e-02$ & 0.07958 \\
\hline & 3 & 0.7893 & $1.979 e-02$ & 0.78945 & $-1.369 e-01$ & 0.0743 & $1.171 e-01$ & $7.45 e-05^{* * *}$ \\
\hline & 4 & 0.8623 & $-1.091 e-01$ & 0.35380 & $-7.304 e-02$ & 0.47754 & $1.822 e-01$ & $0.00500^{* *}$ \\
\hline & Total & 0.825 & $6.663 e-02$ & $0.00559^{* *}$ & $-1.055 e-01$ & $5.7 e-05^{* * *}$ & $3.885 e-02$ & $3.41 e-06^{* * *}$ \\
\hline \multirow{5}{*}{ Thigh BMD } & 1 & 0.7791 & $-4.586 e-03$ & 0.96242 & $-1.205 e-01$ & 0.26162 & $1.251 e-01$ & $0.01704^{*}$ \\
\hline & 2 & 0.8446 & $5.854 e-03$ & 0.9121 & $-8.195 e-02$ & 0.160 & $7.609 e-02$ & $0.000112^{* * *}$ \\
\hline & 3 & 0.8674 & $1.285 e-01$ & 0.262345 & $-3.109 e-01$ & $0.009794^{* *}$ & $1.823 e-01$ & $6.2 e-05^{* * *}$ \\
\hline & 4 & 0.8265 & $-3.097 e-01$ & 0.15855 & $-5.188 e-02$ & 0.78379 & $3.616 e-01$ & $0.00282^{* *}$ \\
\hline & Total & 0.8241 & $3.416 e-02$ & 0.440418 & $-1.062 e-01$ & $0.027139^{*}$ & $7.199 e-02$ & $3.51 e-06^{* * *}$ \\
\hline \multirow{5}{*}{ Trunk BMD } & 1 & 0.701 & $1.207 e-01$ & $0.042169^{*}$ & $-2.042 e-01$ & $0.002757^{* *}$ & $8.352 e-02$ & $0.007302^{* *}$ \\
\hline & 2 & 0.841 & $8.352 e-02$ & $0.00574^{* *}$ & $-1.290 e-01$ & $0.000124^{* * *}$ & $4.546 e-02$ & $4.21 e-05^{* * *}$ \\
\hline & 3 & 0.8457 & $1.698 e-01$ & $0.0250^{*}$ & $-2.922 e-01$ & $0.000299^{* * *}$ & $1.224 e-01$ & $3.52 e-05^{* * *}$ \\
\hline & 4 & 0.9355 & $-2.310 e-02$ & 0.781200 & $-1.551 e-01$ & $0.04079^{*}$ & $1.782 e-01$ & $0.00026^{* * *}$ \\
\hline & Total & 0.8289 & $9.321 e-02$ & $0.00021^{* * *}$ & $-1.529 e-01$ & $3.24 e-08^{* * *}$ & $5.967 e-02$ & $2.17 e-11^{* * *}$ \\
\hline \multirow{5}{*}{ Pelvic BMD } & 1 & 0.7396 & $1.429 e-01$ & 0.102481 & $-2.938 e-01$ & $0.00378^{* *}$ & $1.509 e-01$ & $0.00168^{* *}$ \\
\hline & 2 & 0.8225 & $1.184 e-01$ & $0.016440^{*}$ & $-1.763 e-01$ & $0.00123^{* *}$ & $5.799 e-02$ & $0.00123^{* *}$ \\
\hline & 3 & 0.8425 & $2.123 e-01$ & 0.053760 & $-3.917 e-01$ & $0.000817^{* * *}$ & $1.793 e-01$ & $3.53 e-05^{* * *}$ \\
\hline & 4 & 0.9082 & $-6.424 e-02$ & 0.643974 & $-1.961 e-01$ & 0.115220 & $2.603 e-01$ & $0.001036^{* *}$ \\
\hline & Total & 0.8068 & $1.341 e-01$ & $0.000846^{* * *}$ & $-2.022 e-01$ & $4.05 e-06^{* * *}$ & $6.808 e-02$ & $1.08 e-06^{* * *}$ \\
\hline
\end{tabular}

Note: 1 - underweight group $(n=29), 2$-normal-weight group $(n=132), 3$-overweight group $(n=48)$, and 4-obesity group $(n=32) ; p<0.05^{*} ; p<0.005^{* *}$ $; p<0.0005^{* * *}$.

pelvis $(p<0.05)$, especially in the normal-weight group. The trend of the accumulation of TF in different BMI groups was worthy of our attention. These trends showed a strong regularity in a different part of BMD. Taking whole-body BMD as an example, there was a positive correlation between TF and BMD $(\beta=7.963 e-02, p=0.03602)$. With the increase of BMI, this protective effect gradually weakened until the obesity group began to show a negative correlation trend $(\beta=-2.051 e-01, p=0.09448)$. There was a significant negative correlation between NTF and most BMD $(p<0.05)$. And the higher FFM was associated with higher BMD $(p<0.05)$.

3.5. Compositional Proportional Substitution Analysis. Based on the compositional multiple regression model, the geometric mean of each component of body compositions was used as the reference value. When the total amount remained the same, Figures 3 and 4 showed that each component of the body compositions was increased by $1 \%$ to $4 \%$ in a "one by one" redistribution manner to analyze its effects on BMD. For girls in the overweight group (Figure 3), when TF substituted $4 \%$ NTF or FFM, the BMD increased by about 0.1 and 0.05 units $(p<0.05)$, respectively. When FFM increased (from $1 \%$ to $4 \%$ ) and NTF decreased, BMD showed a downward trend, approximately 0.5 units $(p<0.05)$.

The redistribution of body composition in boys was different from girls (Figure 4). The proportion of TF increased from $1 \%$ to $4 \%$, corresponding reduction in NTF or FFM by $1 \%-4 \%$; the BMD showed an upward trend in the underweight, normal-weight, and overweight groups, especially in the normal-weight group, about 0.08, 0.04, units $(p<0.05)$, respectively. As for the obesity group, though there was no statistical significance, the higher proportion of TF related to the lower BMD. When NTF was substituted by $4 \%$ FFM, BMD increased about $0.05,0.07$ units in the normal-weight and overweight groups. 


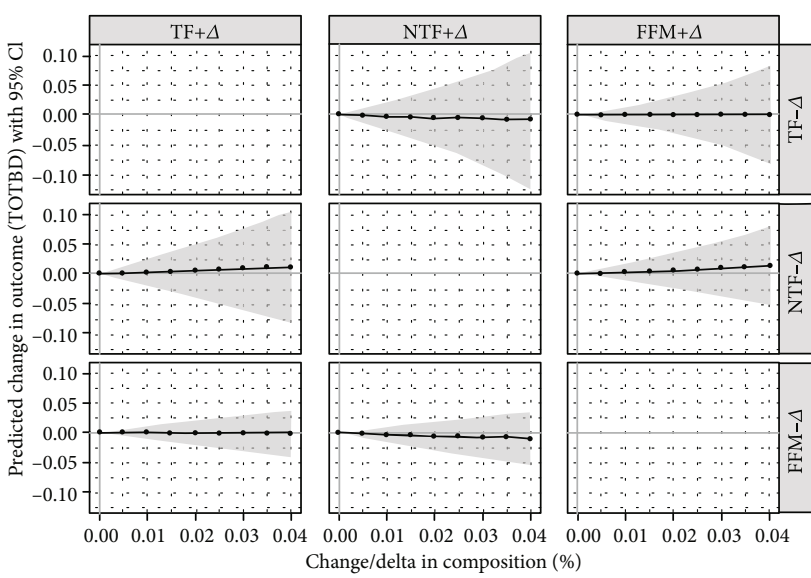

(a)

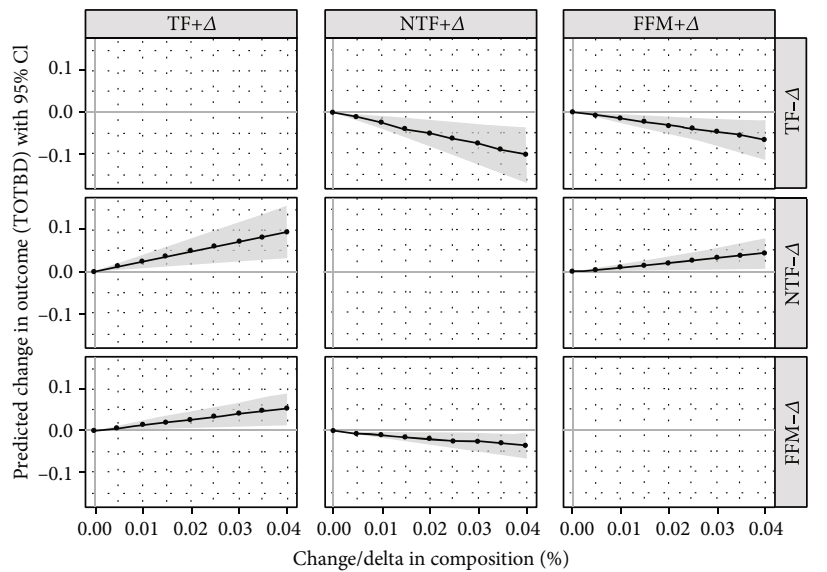

(c)

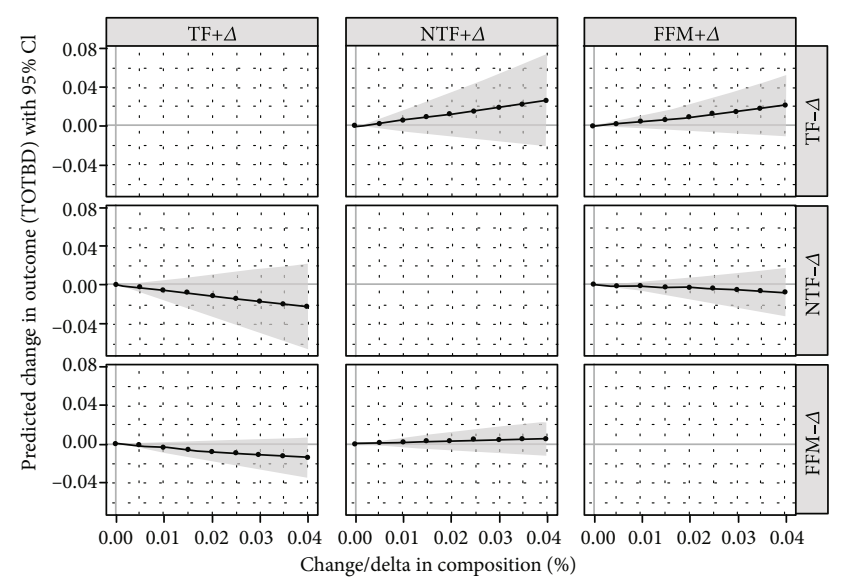

(b)

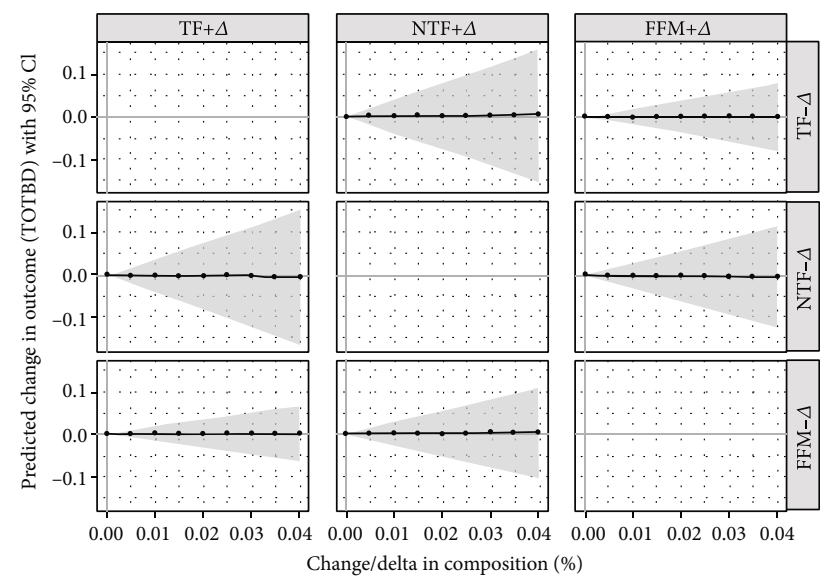

(d)

FiguRE 3: The results of compositional substitution analysis—girls ((a) underweight group; (b) normal-weight group; (c) overweight group; (d) obesity group); BMD: bone mineral density.

\section{Discussion}

This is the first study to examine the association between body composition and BMD using CoDA (ILR transformation), especially in children and adolescents. This method considered the influence of each part of the body compositions on BMD and effectively avoided the problem of multicollinearity. In our study, subjects with a higher percentage of FFM had higher BMD, especially for boys. The association between NTF and most BMD was negative. TF was positively associated with BMD in an overweight group in girls and a normal-weight group in boys. Although there was no statistical significance between TF and BMD in the obese group of boys, the excess TF accumulation related to the lower BMD. In addition, this study excluded the influence of body mass on BMD, which will provide a better explanation for the relationship between body compositions and BMD.

Body compositions are typically divided into different components in different studies. Fat mass is often used as a factor affecting health. In this research, the larger variation matrix value between fat mass and FFM further proved that fat mass was often used as an independent factor to explore the relationship between it and health-related indicators. However, changes in fat mass also affect other components. It can be found from the ternary diagram that as age and weight increased, children and adolescents tended to accumulate more TF and less NTF. In a study of 1007 children aged 6 to 15 in Beijing, Li et al. reported that the area of visceral fat would increase with age [23]. Thus, it is very necessary to discuss body composition by gender during this period. In addition, to avoid the influence between the components, Henriksson et al. and Hetherington et al. made mutual corrections for the quality of fat mass and FFM in their studies [24, 25]. Compared with this method, ILR transformation is better to avoid multicollinearity between multiple components [16]. Therefore, in our study, the CoDA (ILR transformation) included all parts of the body composition in the same model according to gender to better understand the relationship between body compositions and BMD.

The concept that higher FFM is always associated with higher BMD has been widely accepted [26]. The results of our study further demonstrate the positive relationship between FFM and BMD in children and adolescents. Because of the common genetic and hormonal determinants 


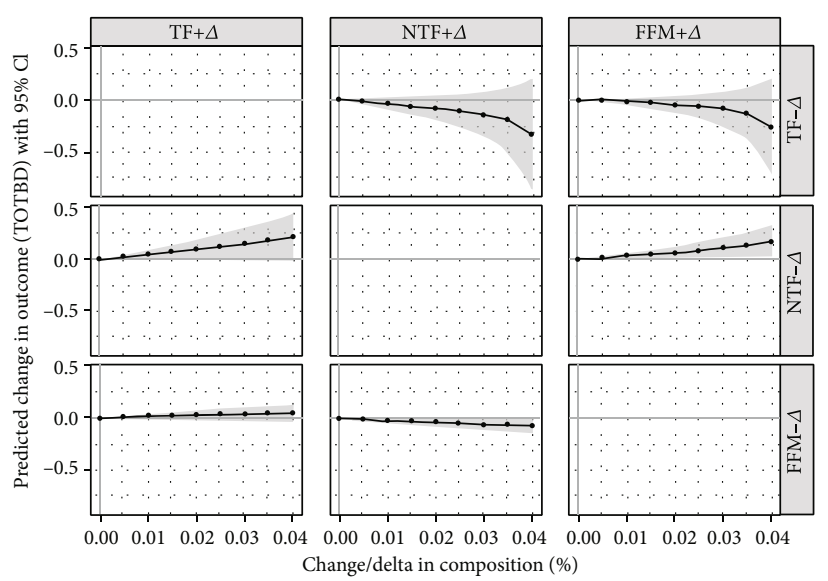

(a)

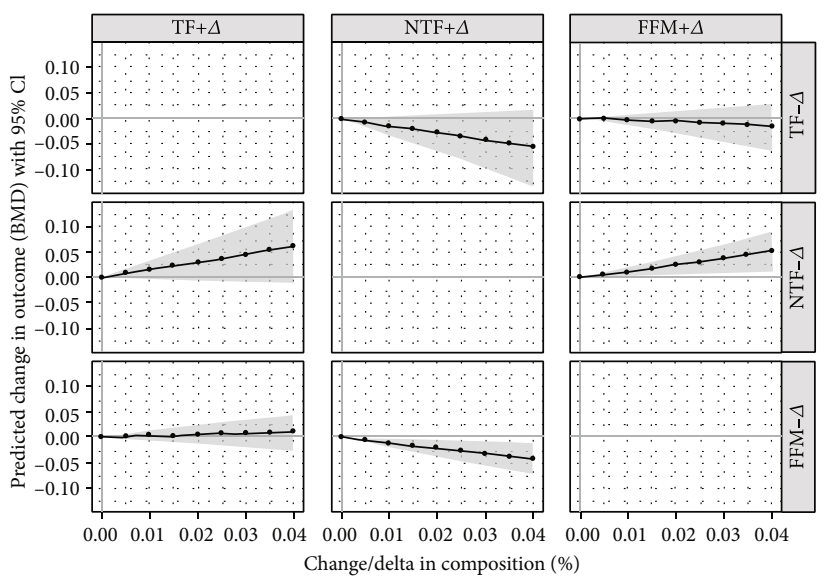

(c)

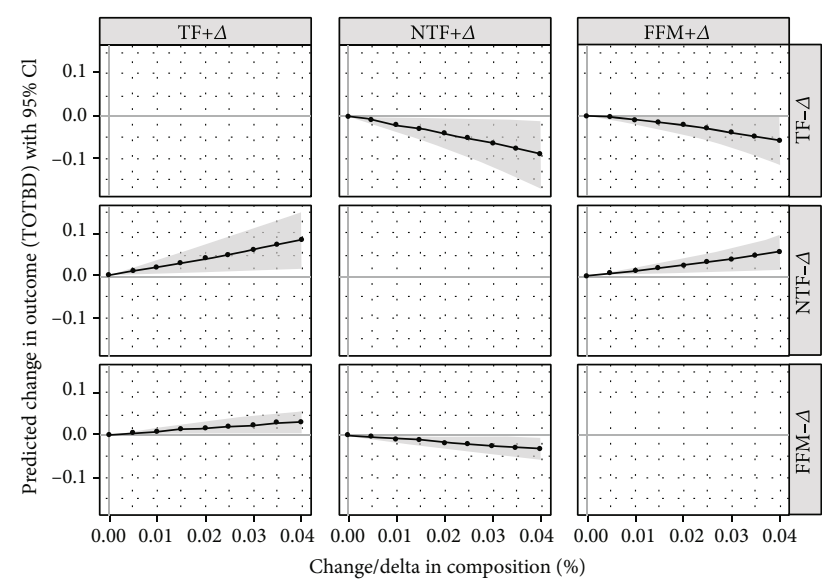

(b)

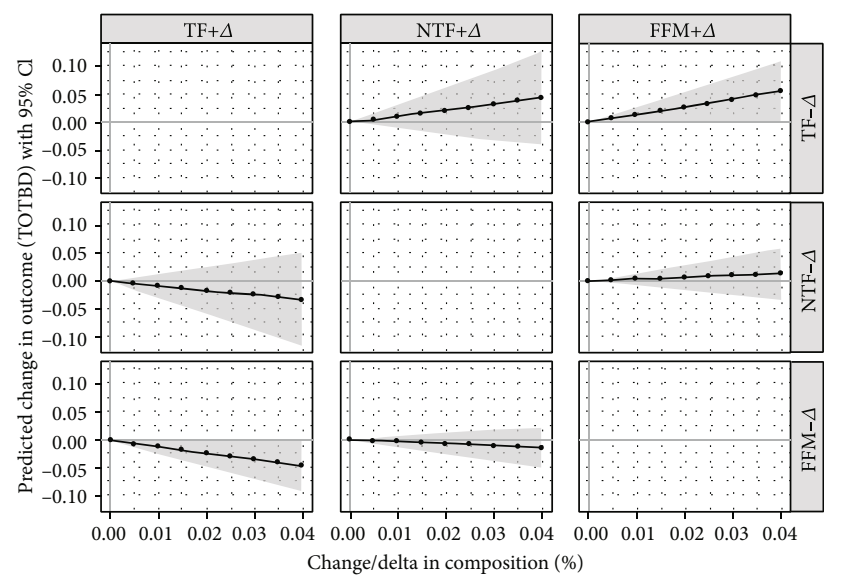

(d)

FIGURE 4: The results of compositional substitution analysis-boys ((a) underweight group; (b) normal-weight group; (c) overweight group; (d) obesity group); BMD: bone mineral density.

$[27,28]$, muscle and bone are closely related adjacent tissues [29], and loss of muscle mass and bone mass throughout life has been proven to be coupled and has been assumed to be part of the same functional unit [26]. In addition, mechanical loading increases bone formation, while weight-bearing exercise improves bone mineral stress [30]. In other words, a higher FFM represents more muscle mass, meaning bones are loaded through muscle action (functional strain), leading to higher BMD [12]. This effect is more significant among boys because boys have far more FFM than girls.

Beyond FFM, the pattern of fat distribution had different effects on BMD. In our research, NTF had a statistically negative correlation with $\mathrm{BMD}$. In the exercise process, the participation of the limbs is crucial; a higher proportion of muscles are needed. Compared with the functional strain produced by muscles on bones, the static strain produced by fat accumulation has a weak effect on bone strength. At the same time, both adipocytes and osteoblasts share a common progenitor. These stem cells are regulated by common factors, such as PPAR- $\gamma$, in the process of differentiation into osteoblasts and adipocytes. Studies have shown that PPAR- $\gamma$ is highly expressed in adipose tissue, making bone tissue more absorptive than bone formation, leading to osteoporosis $[13,31]$.
TF was positively correlated with BMD in the overweight group in girls and the normal-weight group in boys. Inconsistent with our finding, the majority of previous studies involving children and adolescents $[8,32,33]$ or adults $[27$, 34] have reported a negative relationship between $\mathrm{TF}$ and BMD. However, unlike our research with a wide range of body and fat mass, most of these studies were conducted in overweight or obese children. Though no significant negative correlation between TF and BMD was found in our study, the downward trends of TF in boys deserved our attention. Starting from normal weight, as BMI increased, this positive correlation became weaker and weaker, and a negative correlation began to appear in the obesity group. Rokoff et al., in their research, pointed out that the association between central adiposity and BMD only exited in children with the highest levels of abdominal fat [8]. Another study analyzed the body fat and bone mass of 377 adolescents aged 10-19 years and found that BF\% negatively affected bone mass in males, and the higher the BF\% was, the lower BMD values in overweight adolescents [35]. Therefore, in children with relatively high-fat content, the higher FM may strongly correlate with lower BMD. Perhaps there was a threshold of TF, after which the individual is more prone to adverse consequences caused by metabolism. 
Children and adolescents are in a rapid period of growth and development, and each part of their body composition will change significantly. Unlike adults' more stable body composition, a certain amount of TF accumulation may have a better stimulating effect on bone health. In addition, gender differences also require attention when analyzing the relationship between body compositions and bone mineral density. Puberty is an important milestone in the development of body composition. Most studies show that the total FM\% of boys increased before the age of 10-12 and then decreased, while girls' increased gradually with age $[36,37]$. Due to the complexity of hormone secretion during this period, boys accumulated more muscle mass and less fat mass and were more sensitive to fat accumulation. Because of less fat mass and higher sensitivity, when the gravity effect caused by fat accumulation is not enough to offset the negative effects caused by the hormone secreted by fat cells, especially abdominal adipose tissue, the adverse effects on bone density will gradually appear [13]. The substitution analysis further verified the above results when relocating body compositions one by one in different groups. Also, it can provide theoretical support for the formulation of precise intervention plans.

This study provided a new methodological perspective for exploring the relationship between body compositions and BMD using CoDA (ILR transformation) in children and adolescents. The compositional proportional substitution analysis can clearly show the effect of fat distribution and body compositions on BMD. We used DXA to measure the body compositions, which can provide accurate data of FFM and fat mass and fat distribution. Also, there are some limitations. First, the overweight and obesity groups of boys and girls are relatively small; future studies should expand the sample size for analysis. Second, we only divide body fat into two parts: TF and NTF. Future research can divide body composition into more parts or other ways (e.g., fat mass, protein, water, and minerals) to deeply explore the relationship between it and BMD. Finally, our crosssectional data cannot confirm cause and effect.

\section{Conclusions}

Our research demonstrates that FFM has a positive effect on BMD in children and adolescents, especially for boys. The risk of NTF accumulation on BMD was greater than that of TF accumulation. Boys are more sensitive to the amount of TF; excessive TF accumulation may be more harmful to the BMD of boys than girls. Our findings highlight the importance of gender-specific and regional fat influences on $\mathrm{BMD}$ and provide a rationale for further exploration of the mechanisms underlying this observed relationship.

\section{Data Availability}

The results of the research will be available on request from interested persons (query: zhangliubsu@163.com) or from the corresponding author. This limitation is related to the principles of personal data protection. The data will be free of sensitive data, including the date of birth (age will be available at the time of the examination), name, and surname (the coded number of the respondent will be available).

\section{Conflicts of Interest}

The authors declare no conflict of interest.

\section{Acknowledgments}

This research was funded by the Education of the National Social Science Foundation of China, grant number ALA190015. The project name is Comprehensive Intervention and Evaluation System of Chinese Students' Physical Fitness and Health.

\section{References}

[1] A. B. Sopher, I. Fennoy, and S. E. Oberfield, "An update on childhood bone health: mineral accrual, assessment and treatment," Current Opinion in Endocrinology, Diabetes, and Obesity, vol. 22, no. 1, pp. 35-40, 2015.

[2] N. H. Golden, S. A. Abrams, and Committee On Nutrition, "Optimizing bone health in children and adolescents," Pediatrics, vol. 134, no. 4, pp. e1229-e1243, 2014.

[3] J. P. Bonjour, T. Chevalley, S. Ferrari, and R. Rizzoli, "The importance and relevance of peak bone mass in the prevalence of osteoporosis," Salud publica de Mexico, vol. 51, 2009.

[4] C. M. Jankowski, P. Wolfe, S. J. Schmiege et al., "Sex-specific effects of dehydroepiandrosterone (DHEA) on bone mineral density and body composition: a pooled analysis of four clinical trials," Clinical Endocrinology, vol. 90, no. 2, pp. 293-300, 2019.

[5] L. T. Ho-Pham, D. Hans, M. C. Doan, L. D. Mai, and T. V. Nguyen, "Genetic determinant of trabecular bone score (TBS) and bone mineral density: a bivariate analysis," Bone, vol. 92, pp. 79-84, 2016.

[6] M. Pekkinen, H. Viljakainen, E. Saarnio, C. Lamberg-Allardt, and O. Mäkitie, "Vitamin D is a major determinant of bone mineral density at school age," PLoS One, vol. 7, no. 7, article e40090, 2012

[7] M. Heidemann, R. Holst, A. J. Schou et al., "The influence of anthropometry and body composition on children's bone health: the childhood health, activity and motor performance school (the CHAMPS) study, Denmark," Calcified Tissue International, vol. 96, no. 2, pp. 97-104, 2015.

[8] L. B. Rokoff, S. L. Rifas-Shiman, K. M. Switkowski et al., "Body composition and bone mineral density in childhood," Bone, vol. 121, pp. 9-15, 2019.

[9] K. Zhu, M. Hunter, A. James, E. M. Lim, and J. P. Walsh, "Associations between body mass index, lean and fat body mass and bone mineral density in middle-aged Australians: the Busselton Healthy Ageing Study," Bone, vol. 74, pp. 146152, 2015.

[10] Y. M. Cheung, A. Joham, S. Marks, and H. Teede, "The obesity paradox: an endocrine perspective," Internal Medicine Journal, vol. 47, no. 7, pp. 727-733, 2017.

[11] Y. H. Liu, Y. Xu, Y. B. Wen et al., "Association of weightadjusted body fat and fat distribution with bone mineral density in middle-aged Chinese adults: a cross-sectional study," PLoS One, vol. 8, no. 5, 2013. 
[12] J. Liang, Y. Chen, J. Zhang et al., "Associations of weightadjusted body fat and fat distribution with bone mineral density in Chinese children aged 6-10 years," International Journal of Environmental Research and Public Health, vol. 17, no. 5, article 1763, 2020.

[13] J. J. Cao, "Effects of obesity on bone metabolism," Journal of Orthopaedic Surgery and Research, vol. 6, no. 1, p. 30, 2011.

[14] N. K. Pollock, P. J. Bernard, B. Gutin, C. L. Davis, H. Zhu, and Y. Dong, "Adolescent obesity, bone mass, and cardiometabolic risk factors," The Journal of Pediatrics, vol. 158, no. 5, pp. 727734, 2011.

[15] B. Yi, C. Y. Wen, Y. L. Sun, and Y. L. Cai, "The effect of fat distribution on bone mineral density in normal subjects," Chinese Journal of Osteoporosis, vol. 18, no. 7, pp. 610-613, 2012.

[16] J. Aitchison, "The statistical analysis of compositional data," Journal of the Royal Statistical Society Series B, Statistical methodology, vol. 44, no. 2, pp. 139-160, 1982.

[17] D. Dumuid, T. E. Stanford, J. A. Martin-Fernández et al., "Compositional data analysis for physical activity, sedentary time and sleep research," Statistical Methods in Medical Research, vol. 27, no. 12, pp. 3726-3738, 2018.

[18] Ž. Pedišić, "Measurement issues and poor adjustments for physical activity and sleep undermine sedentary behaviour research-the focus should shift to the balance between sleep, sedentary behaviour, standing and activity," Kinesiology, vol. 46, no. 1, 2014.

[19] J. J. Egozcue, V. Pawlowsky-Glahn, G. Mateu-Figueras, and C. Barceló-Vidal, "Isometric logratio transformations for compositional data analysis," Mathematical Geology, vol. 35, no. 3, pp. 279-300, 2003.

[20] Group of China Obesity Task Group, "Body mass index reference norm for screening overweight and obesity in Chinese children and adolescents," Chinese Journal of Epidemiology, vol. 25, no. 2, pp. 10-15, 2004.

[21] Institute of Child and Adolescent Health, Peking University, "Screening standard for malnutrition of school-age children and adolescents," Chinese Journal of School Health, vol. 35, no. 8, article 1266, 2014.

[22] V. Pawlowsky-Glahn and J. J. Egozcue, "Geometric approach to statistical analysis on the simplex," Stochastic Environmental Research and Risk Assessment, vol. 15, no. 5, pp. 384-398, 2001.

[23] X. T. Li, H. Liu, Q. Jiang, L. Yang, and Q. N. Gao, “Characteristic analysis of growth and development of body composition in 6-15 years old children in Haidian district of Beijing," Chinese Journal of Child Health Care, vol. 25, no. 10, pp. 9981001, 2017.

[24] M. Hetherington-Rauth, J. W. Bea, R. M. Blew et al., "Relative contributions of lean and fat mass to bone strength in young Hispanic and non-Hispanic girls," Bone, vol. 113, pp. 144150, 2018.

[25] P. Henriksson, C. Cadenas-Sanchez, M. H. Leppänen et al., "Associations of fat mass and fat-free mass with physical fitness in 4-year-old children: results from the MINISTOP trial," Nutrients, vol. 8, no. 8, p. 473, 2016.

[26] O. Fricke and E. Schoenau, "The 'Functional Muscle-Bone Unit': probing the relevance of mechanical signals for bone development in children and adolescents," Growth Hormone \& IGF Research, vol. 17, no. 1, pp. 1-9, 2007.

[27] Z. Xiao and H. Xu, "Gender-specific body composition relationships between adipose tissue distribution and peak bone mineral density in young Chinese adults," BioMed Research International, vol. 2020, Article ID 6724749, 8 pages, 2020.

[28] V. Singhal, G. D. Maffazioli, N. Cano Sokoloff et al., "Regional fat depots and their relationship to bone density and microarchitecture in young oligo-amenorrheic athletes," Bone, vol. 77, pp. 83-90, 2015.

[29] D. J. DiGirolamo, D. P. Kiel, and K. A. Esser, "Bone and skeletal muscle: neighbors with close ties," Journal of Bone and Mineral Research, vol. 28, no. 7, pp. 1509-1518, 2013.

[30] K. Hind and M. Burrows, "Weight-bearing exercise and bone mineral accrual in children and adolescents: a review of controlled trials," Bone, vol. 40, no. 1, pp. 14-27, 2007.

[31] M. F. Pittenger, A. M. Mackay, S. C. Beck et al., "Multilineage potential of adult human mesenchymal stem cells," Science, vol. 284, no. 5411, pp. 143-147, 1999.

[32] I. F. Júnior, J. R. Cardoso, D. G. Christofaro, J. S. Codogno, A. C. de Moraes, and R. A. Fernandes, "The relationship between visceral fat thickness and bone mineral density in sedentary obese children and adolescents," BMC Pediatrics, vol. 13, no. 1, p. 37, 2013.

[33] N. K. Pollock, P. J. Bernard, K. Wenger et al., "Lower bone mass in prepubertal overweight children with prediabetes," Journal of Bone and Mineral Research, vol. 25, no. 12, pp. 2760-2769, 2010.

[34] X. Fu, X. Ma, H. Lu, W. He, Z. Wang, and S. Zhu, “Associations of fat mass and fat distribution with bone mineral density in pre- and postmenopausal Chinese women," Osteoporosis International, vol. 22, no. 1, pp. 113-119, 2011.

[35] L. N. Mosca, T. B. Goldberg, V. N. da Silva et al., "Excess body fat negatively affects bone mass in adolescents," Nutrition, vol. 30, no. 7-8, pp. 847-852, 2014.

[36] B. Guo, Q. Wu, J. Gong et al., "Gender difference in body fat for healthy Chinese children and adolescents," Childhood Obesity, vol. 12, no. 2, pp. 144-154, 2016.

[37] L. Cheng, H. Pohlabeln, W. Ahrens et al., "Sex differences in the longitudinal associations between body composition and bone stiffness index in European children and adolescents," Bone, vol. 131, article 115162, 2020. 